\title{
Internet das coisas em bibliotecas: proposta de um sistema para monitoramento de ruído para bibliotecas
}

\author{
Fernanda Vasconcelos Amaral \\ Mestre; Universidade Federal de Lavras, Lavras, MG, Brasil; \\ fernandav.br@gmail.com; ORCID: https://orcid.org/0000-0001-6197-9548 \\ Jordan Paulesky Juliani \\ Doutor; Universidade do Estado de Santa Catarina, Florianópolis, SC, Brasil; \\ jordanjuliani@gmail.com; ORCID: https://orcid.org/0000-0001-7823-6644 \\ Raphael Winckler de Bettio \\ Doutor; Universidade Federal de Lavras, Lavras, MG, Brasil; \\ raphaelwb @ufla.br; ORCID: https://orcid.org/0000-0003-3635-3580
}

\begin{abstract}
Resumo: As bibliotecas são unidades de informação que estão constantemente adotando novos recursos tecnológicos para melhorar a qualidade da sua oferta de produtos e serviços. Atualmente, uma tecnologia proeminente é a internet das coisas (IoT), que promete grandes transformações na sociedade ao proporcionar uma maior integração entre os mundos físico e digital. Por isso, este estudo objetiva discutir a possibilidade de implementação da internet das coisas em uma biblioteca universitária ao investigar demandas dos usuários que poderiam ser sanadas por meio dessa tecnologia. Trata-se de uma pesquisa aplicada, caracterizada como exploratória e descritiva. A metodologia adotada é um estudo de caso na Biblioteca Universitária da Universidade de Lavras, fundamentada na engenharia de requisitos e no design thinking. $\mathrm{O}$ resultado final constitui-se na idealização de um sistema de monitoramento de ruídos, baseado em IoT, com a finalidade de contribuir na manutenção do silêncio nas áreas de estudo e na utilização do ruído como um dado para apoiar a gestão da biblioteca.
\end{abstract}

Palavras-chave: Internet das Coisas; Sistema de Monitoramento de Ruídos; Gestão de Bibliotecas; Bibliotecas Inteligentes

\section{Introdução}

O avanço da tecnologia no cotidiano da sociedade está modificando profundamente a forma como o ser humano trabalha, realiza suas atividades pessoais e até mesmo interage uns com os outros. Seja para viabilizar, facilitar ou agilizar o desempenho de uma tarefa, as tecnologias estão cada vez mais presentes como um intermediário entre o sujeito e o ambiente que o rodeia. 
O ambiente das bibliotecas não ficou indiferente a esses avanços tecnológicos. Da substituição das fichas catalográficas por sistemas eletrônicos de gerenciamento de acervo, passando pela adoção das máquinas de autoempréstimo e autodevolução, as bibliotecas incorporaram vários recursos tecnológicos em suas rotinas de trabalho.

Contudo, mais do que informatizar as bibliotecas, é necessário também criar novos tipos de serviços, ainda indisponíveis ao público atendido. Nesta missão, as novas tecnologias da $4^{\mathrm{a}}$ Revolução Industrial são importantes aliadas para propiciar experiências inovadoras, que dinamizem e facilitem a utilização dos serviços da biblioteca, aumentando, consequentemente, o nível de satisfação dos usuários atendidos. Ao melhorar a experiência do usuário, as bibliotecas serão capazes de manter a fidelidade dos usuários atuais, que continuarão a frequentar o local, além de atrair um novo público em potencial e fortalecer sua imagem institucional perante a comunidade atendida diante da excelência dos serviços prestados.

Entre as diversas oportunidades para inovar em bibliotecas, a internet das coisas (IoT) destaca-se por possibilitar que os espaços físicos se tornem mais interativos e os serviços ofertados mais personalizados (HAHN, 2017), visto que a IoT possui a capacidade de promover a criação de novos serviços e a melhoria das condições do ambiente físico e dos serviços já prestados pela biblioteca.

Por isso, foi desenvolvido um estudo na Biblioteca Universitária da Universidade Federal de Lavras (BU/UFLA) com o objetivo de investigar se os serviços prestados por essa unidade de informação poderiam ser melhorados por meio da tecnologia de IoT. Os procedimentos metodológicos envolveram a utilização de engenharia de requisitos e técnicas de design thinking para compreender as demandas e problemas enfrentados pelos usuários na BU/UFLA e a viabilidade de implementar soluções baseadas em IoT. Ao final da coleta e da análise de dados, optou-se por desenvolver um sistema de monitoramento de ruído para medir a intensidade de ruídos nos espaços de estudo da biblioteca, por meio da detecção e monitoramento de sinais sonoros no ambiente. Com isso, utilizou-se o ruído como dado para apoiar as atividades de gestão da biblioteca. 
Nas próximas seções, apresenta-se uma revisão bibliográfica sobre a IoT, com foco em seu conceito e arquitetura tecnológica. Em seguida, o percurso metodológico percorrido é detalhado. Na quarta seção, apresenta-se em detalhes a proposta do sistema de monitoramento de ruídos. Por fim, faz-se as considerações finais e sugestões de trabalhos futuros.

\section{Internet das coisas}

A primeira menção ao termo IoT ocorreu no ano de 1999, quando o pesquisador britânico do Massachusetts Institute of Technology (MIT) Kevin Ashton proferia uma palestra em um evento sobre a tecnologia de identificação por rádio frequência (RFID) (ASHTON, 2009). Existem diversas definições de IoT, mas, em síntese, a IoT conecta objetos físicos à internet mediante a utilização de sensores, atuadores, transceptores e microcontroladores, de forma a permitir que objetos analógicos sejam capazes de coletar e transmitir dados em rede (ATZORI; IERA; MORABITO, 2010; ZANELLA et al., 2014). Sob um ponto de vista menos técnico, a IoT

[...] é um conceito que propõe literalmente a interconexão via Internet de todos os objetos existentes, as chamadas 'coisas'. Esta interconexão possibilitaria que os objetos 'conversassem' entre si e 'tomassem decisões' sem a intervenção humana (DUTRA; TORIANI, 2016, p. 86).

Portanto, a IoT pode ser entendida como uma tecnologia que transforma entidades analógicas em digitais, gerando um alto valor agregado para produtos e serviços, sem a necessidade inerente e constante da intervenção humana.

De acordo com Shin (2010), o entendimento de objeto (coisa) é bastante amplo e abrange tanto artefatos desenvolvidos pelo homem, como um automóvel por exemplo, como também o próprio ser humano ou outros animais. A transformação de seres vivos em uma entidade digital conectada à internet pode ser realizada por meio de implantes de biochips ou por meio de tecnologias menos invasivas como pulseiras ou relógios inteligentes, por exemplo.

A conexão direta dos objetos físicos à internet configura-se como um passo primordial para a inserção completa da realidade física no ciberespaço. Se a internet, inicialmente, propiciou a conexão em rede dos computadores, seguida 
pela conexão entre as pessoas, por meio de recursos como e-mail, chats e redes sociais, com IoT os objetos e ambientes também são conectados em rede para ampliar as suas funcionalidades por meio da captação, transmissão e processamento de dados (ASHTON, 2009). Essa hiperconexão contribuirá para gerar inúmeras soluções para problemas presentes na sociedade, além de melhorar os serviços e produtos já existentes, trazendo mais comodidade e qualidade de vida para os cidadãos.

A despeito do nome focar no termo "coisa", não são os objetos em si que são inteligentes na IoT, mas $\operatorname{sim}$ as plataformas, que conectam "coisas analógicas" com um sistema digital (ONLINE COMPUTER LIBRARY CENTER, 2015). Para isso, é necessário criar uma arquitetura tecnológica com várias camadas que proporcionem o funcionamento da IoT. Essa arquitetura é ilustrada na Figura 1 e detalhada em seguida.

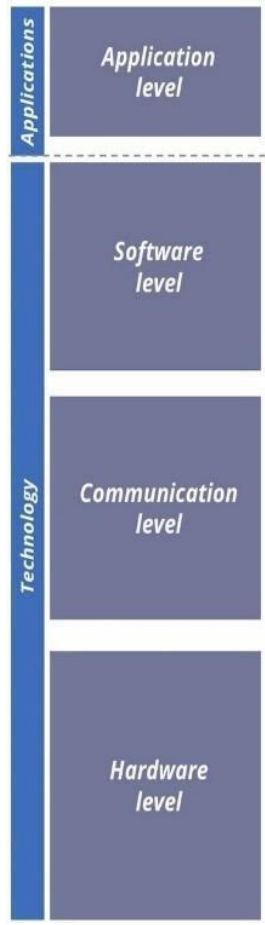

Figura 1 - Níveis de arquitetura de sistemas de IoT.

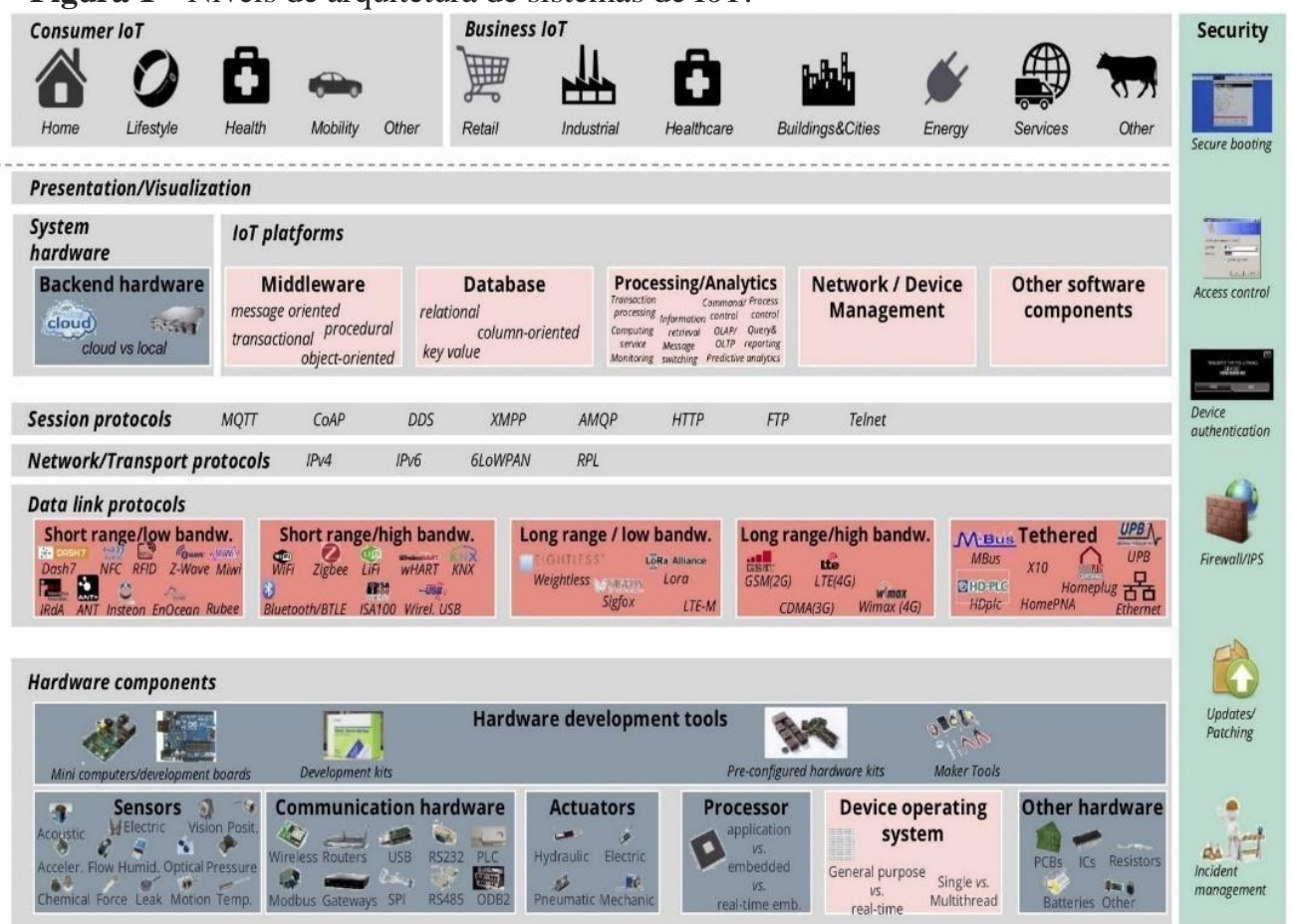

Fonte: Columbus (2019).

A arquitetura tecnológica por trás de um sistema de IoT é bastante variável e utiliza um vasto número de tecnologias e camadas de acordo com a especificação de cada sistema. A empresa de consultoria IoT Analytcs GmbH propõe uma arquitetura baseada em quatro níveis principais: hardware, 
comunicação, software e aplicação final (SCULLY, 2019). Esses quatro níveis estão permeados por camada fundamentada na segurança de dados.

Nomeado por alguns autores (DOMINGO, 2012; KHAN et al., 2012) como camada de percepção, o nível de hardware identifica o objeto e coleta informações. É composto por sensores, dispositivos de rede, atuadores (dispositivo que produz movimento ao receber energia elétrica, mecânica ou hidráulica), processadores, microchips, placa de processamento, e outros dispositivos físicos. Há um amplo número de equipamentos que podem ser utilizados, sejam opções já formatadas e disponíveis comercialmente, sejam alternativas criadas por meio de kits e ferramentas de desenvolvimento que permitem uma construção própria e customizada dos equipamentos.

Indicada como camada de rede por outros autores (DOMINGO, 2012; KHAN et al., 2012), o nível de comunicação transmite os dados recebidos do hardware por meio de redes com fio ou sem fio, sendo, portanto, responsável pelo tráfego de dados. Os protocolos de comunicação podem ser proprietários ou de fonte aberta (SCULLY, 2019) e estão divididos em três tipos: protocolos de enlace de dados ou link de dados, protocolos de transporte e rede, e protocolos de sessão.

No nível de software, também denominado de camada de middleware (KHAN et al., 2012), os dados transmitidos são armazenados em um banco de dados, processados, analisados e transformados para serem utilizados em uma aplicação. O sistema pode ser construído tanto em uma rede local como ser armazenado nas nuvens. No nível do software, há duas camadas, o back-end, constituído pelos componentes computacionais responsáveis pelas regras de funcionamento do sistema, e o front-end, que é a interface mais amigável a ser visualizada pelo usuário final do sistema.

Por fim, no nível de aplicação, ocorre o gerenciamento da aplicação junto ao usuário final do sistema, que pode ser um consumidor individual ou um consumidor institucional. Essa aplicação deve gerar as ações necessárias para atender as necessidades do usuário final.

Esses quatro níveis interagem para propiciar o funcionamento satisfatório do sistema de IoT e devem ser construídos considerando padrões de 
segurança satisfatórios. Nesse quesito, devem ser consideradas configurações contra invasões de malwares, criptografia de mensagens e de disco, autenticação do usuário, controle de acesso, gerenciamento de incidentes com os equipamentos e dispositivos utilizados, entre outras abordagens.

Em síntese, para que um sistema de IoT seja construído há uma confluência de várias tecnologias, dispostas em camadas que interagem entre si para entregar uma solução para o cliente final. A estruturação dessas camadas e utilização de tecnologias irá variar em grande medida de acordo com a funcionalidade e especificidade de cada sistema de IoT.

\section{Procedimentos metodológicos}

Em relação ao objetivo, a presente pesquisa caracteriza-se como exploratória e descritiva, com o objetivo de apresentar e descrever a proposta de um sistema baseado em IoT para bibliotecas. Quanto à sua finalidade, trata-se de uma pesquisa aplicada, com o propósito de resolver um problema específico e ter uma aplicação prática dentro do ambiente das bibliotecas.

O método escolhido é o estudo de caso, por oferecer a oportunidade de investigar empiricamente e em detalhes as necessidades dos usuários de uma biblioteca real e propor um sistema customizado de acordo com as demandas e limitações identificadas no contexto em estudo.

Para solucionar o problema da pesquisa e alcançar os objetivos propostos, foi adotado um procedimento metodológico fundamentado na engenharia de requisitos, composto por três etapas: fase de elicitação de requisitos, fase de especificação de requisitos e fase de validação de requisitos.

A fase de elicitação de requisitos da pesquisa objetivou coletar dados que fundamentassem a construção do sistema de IoT. Essa etapa envolveu dois grupos de atividades: a pesquisa com os usuários da BU/UFLA, realizada com 7 participantes em dezembro de 2019; e a pesquisa com os gestores da BU/UFLA, realizada com 6 participantes em fevereiro de 2020. Em ambas etapas foram utilizadas técnicas de design thinking. Na primeira etapa, com os usuários, utilizou-se as técnicas de questionário online, brainstorming, caderno de sensibilização e grupos focais para entender a relação dos usuários com a 
biblioteca, identificar suas demandas não atendidas e discutir possíveis soluções baseadas em IoT que poderiam ser implementadas para melhorar os serviços oferecidos pela biblioteca. Na segunda etapa, com os gestores, utilizou-se as técnicas de entrevista, brainstorming, cardápio de ideias, cardsorting e grupo focal para consolidar as propostas de soluções geradas nas dinâmicas com os usuários e estabelecer qual proposta seria prioritária para ser implementada, além de ser viável financeira e tecnologicamente.

Após todas as análises dos dados coletados na fase de elicitação de requisitos, optou-se por desenvolver um sistema de monitoramento de ruídos para tornar as condições das áreas de estudo da BU mais satisfatórias. Essa proposta foi escolhida por ter sido identificado que o barulho dentro da BU/UFLA foi um problema bastante citado pelos usuários e, do ponto de vista da viabilidade, a criação de um sistema de monitoramento de barulho possui uma complexidade e custos menores que as outras propostas consideradas prioritárias. Foi iniciada então a ideação desse sistema.

Destacamos que, embora a área de Biblioteconomia atualmente preconize a criação de bibliotecas que ofereçam espaço para interação e comunicação entre os usuários (MACWHINNIE, 2003), a Biblioteca da UFLA apresenta um grande fluxo de usuários, o que pode ocasionar excesso de ruído em determinados horários do dia. A intenção do sistema a ser criado é auxiliar o bibliotecário a monitorar quando esses excessos ocorrem, pois mesmo com sucessivas ampliações do espaço físico da biblioteca, com a criação de espaços específicos para estudo em grupo e individual, ainda persistem dificuldades relacionados a usuários conversando em tom de voz muito elevado ou não se atentando que na área de estudo individual deve prevalecer o mínimo de barulho possível.

$\mathrm{Na}$ fase de especificação de requisitos da pesquisa, os requisitos do sistema de monitoramento de ruído foram detalhados e especificados em um formato padrão compreensível tanto pela equipe de bibliotecários e usuários quanto pelos desenvolvedores da área de Ciência da Computação. Foi elaborado um Documento de Requisitos de Sistema (DRS), de acordo com os parâmetros da norma IEEE 830/1998 (INSTITUTE OF ELECTRICAL AND 
ELECTRONICS ENGINEERS, 1998), para reunir as informações necessárias para a criação do sistema de IoT.

Para a determinação de sua eficiência e eficácia, foi prevista por esta pesquisa a aplicação de Prova de Conceito (PoC - Proof of Concept). Para Andrade, Arakaki e Becerra (2006, p. 291) "Define-se prova de conceito como uma técnica que permite demonstrar que uma determinada ideia é tecnicamente possível [...]”. Dessa forma, foi testado se a solução tecnológica proposta teoricamente poderia funcionar na prática. Ressaltamos que o objetivo do PoC é testar a viabilidade técnica do sistema, sem ainda validá-lo como uma solução pronta. Por isso não foram feitos testes para comparar a qualidade de captura do som do aparelho celular em relação ao um medidor profissional, sendo essa etapa algo a ser realizado em estudo futuro.

Na fase de avaliação, o DRS e PoC foram analisados pelos gestores e pelo profissional de TI da BU/UFLA por meio de um grupo focal. Em seguida, o DRS foi avaliado também pelo mesmo grupo de usuários que participou das dinâmicas de design thinking na etapa de elicitação de requisitos. O objetivo dessa avaliação foi para que os participantes apontassem omissões de dados, requisitos incompletos ou mal formulados, possíveis contradições na especificação dos requisitos, entre outras informações que auxiliassem a melhorar o sistema proposto. Na próxima seção serão apresentadas a proposta e a avaliação do sistema de monitoramento de ruídos idealizado ao final da pesquisa.

O método de análise escolhido para tratar os dados quantitativos foi a estatística descritiva, que abrange um conjunto de procedimentos e técnicas para organizar, sintetizar e descrever dados, por meio de quadros, gráficos, tabelas e indicadores numéricos (REIS, 2008; SANTOS, 2018). Utilizou-se em especial a distribuição de frequências, que "[...] compreende a organização dos dados de acordo com as ocorrências dos diferentes resultados observados [...]" (BARBETTA, 2002, p. 14), ou seja, indica a quantidade ou porcentagem da ocorrência de determinada classe de dados observados.

Referente aos dados qualitativos, provenientes das questões abertas do questionário online, da sessão de brainstorming, do caderno de sensibilização, 
dos grupos focais e das entrevistas, adotou-se a análise de conteúdo como técnica de tratamentos dos dados coletados. Optou-se pela análise de conteúdo por ser um método propício para observar nos indivíduos ou grupos de pessoas as suas opiniões, satisfações, insatisfações e a natureza do problema em estudo (FREITAS; JANISSEK, 2000).

\section{Sistema para monitoramento de ruídos}

Terminada a fase de elicitação, iniciou-se a especificação dos requisitos necessários para a criação do sistema de monitoramento de ruídos. Foram elaborados o Documento de Requisitos de Sistema e a Prova de Conceito para demonstrar teoricamente como o sistema funcionará após sua implementação na prática. A seguir apresenta-se a proposta com as especificações do sistema e a avaliação do DRS e do PoC pelos gestores, um profissional de TI e os usuários da BU/UFLA.

\subsection{Sistema proposto}

Nos resultados referentes à experiência de usuário ao utilizar os espaços de estudo da BU, os usuários apontaram que valorizam um espaço de estudo silencioso para aumentar sua capacidade de foco e concentração. De fato, Servilha e Delatti (2014) apontam que um ambiente acústico inadequado interfere no processo de aprendizagem ao provocar distrações e irritabilidade nos alunos, enquanto Cândido e Jucá (2017) afirmam que o excesso de ruído reduz a produtividade e propicia erros. Ademais, indivíduos expostos a níveis intensos de ruído por período prolongado apresentam maior prevalência de hipertensão arterial e doenças cardiovasculares (ROCHA et al., 2002).

No Brasil, a NBR 10152, publicada pela Associação Brasileira de Normas Técnicas (ABNT) primeiramente em 2017 e corrigida em 2020, estabelece níveis aceitáveis de ruídos em ambientes externos e internos às edificações. A norma recomenda que as bibliotecas tenham níveis de ruído ambiente entre 35 a 45 decibels $(\mathrm{dB})$ para manter o conforto acústico do local. Entende-se conforto acústico como o nível de ruído aceitável para cada ambiente, de forma a não impactar na saúde e produtividade dos usuários. Já 
ruídos acima de $85 \mathrm{~dB}$ são capazes de provocar danos à saúde dos indivíduos, de acordo com a Administração de Segurança e Saúde Ocupacional dos Estados Unidos (OCCUPATIONAL SAFETY AND HEALTH ADMINISTRATION, 1996).

Em pesquisas práticas de medição de ruídos em bibliotecas de Instituições de Ensino Superior, foram constatados níveis elevados de ruído nesses ambientes, tal como descrito pelos usuários da BU/UFLA. Pereira, Silva e Sales (2011), em pesquisa no Maranhão, obtiveram uma variação na intensidade de barulho entre 55 e 66 dB. Botari et al. (2017) encontraram uma variação entre 61 e $73 \mathrm{~dB}$ em uma biblioteca no estado do Paraná. E Silva, Almeida e Albuquerque Neto (2017), em estudo no Piauí, obtiveram um resultado ainda mais elevado que a recomendação da $\mathrm{ABNT}$, com uma variação no nível de ruído entre 67 e $80 \mathrm{~dB}$.

Para solucionar essa demanda, foi proposto a criação de um sistema, denominado $\mathrm{Xi}$, para monitorar a intensidade de ruídos nas mesas de estudos da BU e auxiliar os bibliotecários a manterem um ambiente adequado para os usuários. O nome $\mathrm{Xi}$ foi escolhido por ser uma onomatopeia para o som que algumas pessoas fazem ao pedir silêncio. Embora a grafia normalmente utilizada seja Shhh, optou-se pela adoção de uma transcrição fonética mais próxima ao som emitido ao se utilizar essa figura de linguagem para pedir silêncio. A função básica do sistema é captar sinais sonoros em torno de cada mesa de estudo em grupo ou individual e emitir um alerta para os usuários e bibliotecários quando o nível de ruído ultrapassar os parâmetros previamente estabelecidos.

O alerta para o usuário será feito por meio de uma luz que acenderá na mesa onde esteja ocorrendo um barulho muito excessivo. Esse sinal luminoso será um incentivo para que os usuários daquela mesa reduzam seu tom de voz para não incomodar os colegas sentados nas mesas adjacentes.

O bibliotecário, por sua vez, receberá o alerta por meio de um aplicativo, com a indicação de qual mesa está com ruído intenso. Desse modo, o bibliotecário pode dirigir-se à mesa em questão e conscientizar os usuários sobre 
a necessidade de respeitar os colegas para que todos tenham um ambiente propício ao estudo.

É importante destacar que a BU/UFLA possui dois tipos de espaços de estudo com características bastante diferenciadas: áreas para estudo individual e áreas para estudo em grupo. A primeira deve primar pelo menor nível possível de ruído, enquanto na segunda haverá uma tolerância maior de barulho devido à necessidade de discussões entre os integrantes do grupo de estudo. Por isso, os parâmetros estabelecidos para disparar o alerta de ruído devem ser diferenciados para cada área de estudo.

Além da captação por mesa, um sistema geral de alerta para o ambiente como um todo também seria eficiente para quando várias mesas ao mesmo tempo estiverem com barulho elevado. Assim poderia haver um alerta sonoro informando que o nível de barulho está muito alto e solicitando que todos os usuários suavizem o tom de voz. Esse sensor geral poderia ser instalado no teto ou nas paredes e ter um raio de alcance maior do que o das mesas.

Em um nível mais avançado, o Xi processará os dados dos sons coletados e criará um mapa de calor com visualização em tempo real das variações de ruídos em cada área de estudo. Para efeito ilustrativo, a Figura 2 apresenta a planta baixa da BU/UFLA com uma simulação indicativa do nível de ruído das áreas de estudo. Esse mapa pode ser criado em um editor de imagem, como o Sketchup, para desenhar mapas mais robustos com modelagem 3D. Ou, uma solução mais sofisticada, seria apresentar o mapa de calor com imagens reais da biblioteca, com visualização similar ao Google Earth Studio, em vez de utilizar uma representação gráfica do ambiente. 
Figura 2 - Representação gráfica do mapa de calor do nível de ruído dentro da biblioteca

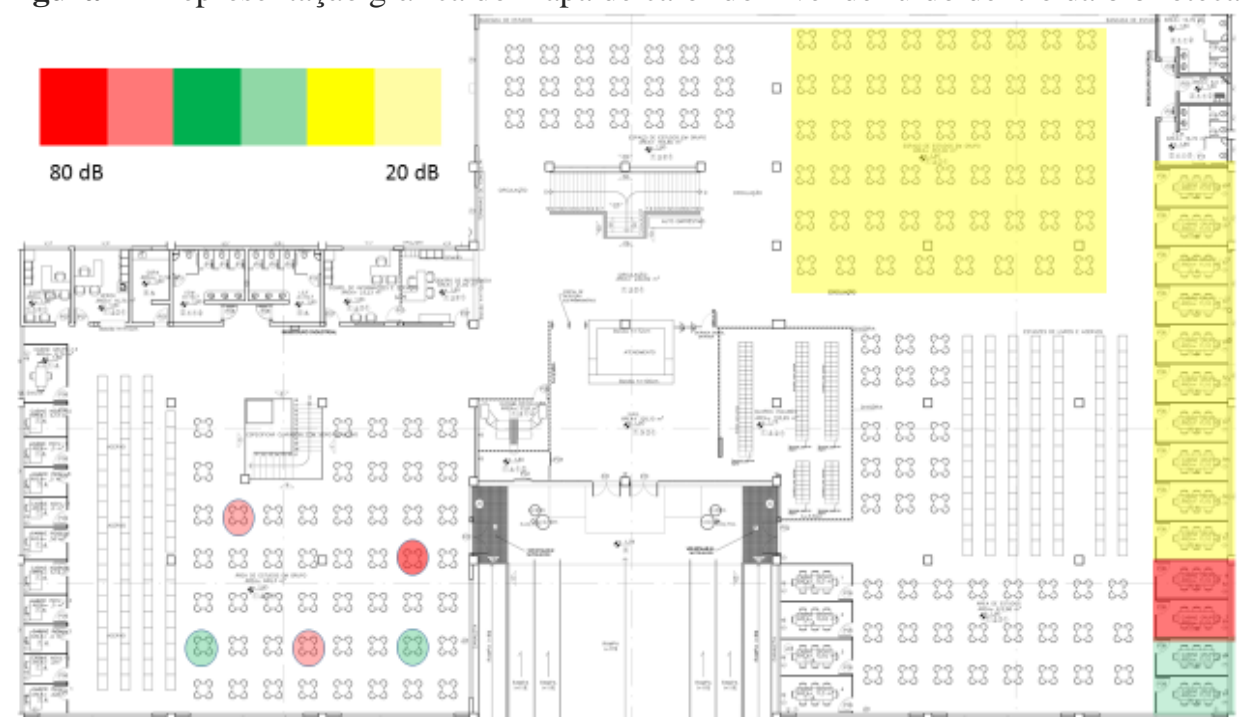

Fonte: Elaborada pela autora (2020).

Outro recurso do nível avançado do sistema, são as funcionalidades de gerar relatórios estatísticos e dashboard (painéis visuais) com informações sobre os dias e horários com maior e menor intensidade de barulho e quais áreas da biblioteca são, geralmente, mais silenciosas.

Após um período com coletas diárias dos sinais sonoros, os dados processados pelo sistema podem servir para medir e compreender a ocupação das áreas de estudo da BU e apoiar as atividades de gestão desenvolvidas. Com base nas informações geradas, os bibliotecários podem planejar mudanças de layout do espaço para que não haja concentração de usuários em determinadas áreas; readequar a distância entre as mesas de estudo; determinar com mais precisão horários e dias com maior movimento de usuários e, portanto, com necessidade de mais servidores trabalhando nesses períodos; identificar a necessidade ou não de realizar campanhas de conscientização sobre a importância do silêncio dentro da BU; entre inúmeras outras possibilidades.

As informações disponibilizadas pelo sistema também auxiliarão os usuários, que podem se planejar para frequentar a BU em dias e horários com picos menores de ruídos e, possivelmente, menos movimentação de pessoas. Ademais, essas informações servem para orientar os usuários sobre quais são os espaços e horários para uso da biblioteca, de acordo com o propósito almejado. Dessa forma, mais do que um sistema para controlar o ruído dentro da 
biblioteca, o Xi utilizará o ruído como dado para desenvolver outras funcionalidades.

Definidas as características e funcionalidades desejáveis do sistema, foi elaborado um DRS, com a especificação dos requisitos do sistema proposto. $\mathrm{O}$ documento foi elaborado em um formato estruturado de acordo com a norma IEEE 830/1998, para ser compreensível tanto pelos usuários do sistema (gestores e usuários da BU) quanto por profissionais da área técnica de TI. Concomitantemente, foi elaborada uma prova de conceito para verificar as possibilidades práticas de implantação do sistema.

\subsection{Criação da Prova de Conceito do sistema}

Inicialmente, pensou-se em construir um dispositivo próprio para captar o ruído do ambiente. Essa alternativa é tecnicamente viável, como demonstram Medeiros et al. (2013), Cândido e Jucá (2017) e Cândido et al. (2018). Contudo, todos os componentes do dispositivo teriam que ser homologados pela Agência Nacional de Telecomunicações (Anatel) e seria necessário construir inúmeros sensores para serem instalados em todas as mesas da BU, demandando tempo e mão de obra disponível.

Para sanar esses empecilhos, optou-se por basear-se a PoC na utilização de aparelhos celulares para captar os ruídos do ambiente, aproveitando os componentes instalados nesses dispositivos, que já foram aprovados pela Anatel previamente para serem comercializados. Dessa forma, toda a complexidade de montar um dispositivo novo e homologar junto a Anatel é descartada.

Outro benefício desta alternativa é trabalhar com um projeto sustentável, ao reaproveitar celulares antigos, que seriam possivelmente inutilizados ou descartados. Os celulares poderiam ser recolhidos como doação voluntária, para abatimento de multas na $\mathrm{BU}$, ou em um projeto em parceria com empresas de eletrônicos.

Considerando a indisponibilidade de tomadas nas mesas de estudo da biblioteca, os celulares serão conectados a um power bank (carregador portátil) para manter os aparelhos em funcionamento. A construção do Xi será estruturada em 4 camadas de arquitetura: 
a) camada de hardware: os celulares acoplados nas mesas de estudo captarão os decibels dos ruídos do ambiente em um raio de alcance em torno dessas mesas;

b) camada de comunicação: os dados coletados serão transmitidos por meio da rede sem fio da biblioteca para um software;

c) camada de software: os dados serão processados e analisados pelo software para operacionalizar as funcionalidades de emissão de alerta, criação do mapa de calor por nível de ruído, emissão de relatórios e criação do dashboard;

d) camada de aplicação: os dados processados serão disponibilizados na interface de acesso livre (para os usuários da biblioteca) e na interface administrativa (para os bibliotecários).

Para configurar o sistema, primeiramente será instalado nos celulares o aplicativo para coletar os decibels dos ruídos do ambiente. Esses celulares serão então instalados nas mesas de estudo e será criado o mapa virtual do ambiente da biblioteca, indicando as coordenadas (latitude e longitude) da localização de cada celular.

Os celulares, conectados à fonte de energia do power bank, captarão os decibels do ruído em torno das mesas e transmitirão para o servidor de processamento de dados. O software receberá e processará esses dados, formando matrizes com os valores dos ruídos em decibels, que serão calculados para indicar no mapa virtual da biblioteca os locais adjacentes de onde efetivamente o ruído foi lido. Esses dados processados podem então ser disponibilizados por meio de aplicativos para celular e desktop.

Inicialmente, os testes técnicos seriam realizados na BU/UFLA, mas, devido à pandemia de COVID-19 no início do ano de 2020, os testes ocorreram na residência de um dos pesquisadores do estudo. O teste alcançou seu objetivo de simular o funcionamento do sistema e verificar a viabilidade da utilização de celulares como coletores de dados de som.

O PoC testou os seguintes procedimentos: o celular realiza a captação dos ruídos do ambiente (Figura 3a) e a cada dez segundos comunica ao servidor 
central o maior ruído que ocorreu no ambiente; se o ruído for abaixo do padrão estabelecido, o servidor apenas armazena o dado; se o ruído for acima do padrão, o servidor armazena o dado e emite um alerta de excesso de ruído (Figura 3b). Após o processamento desses dados, o servidor desenha o mapa de calor baseado nos ruídos (Figura 3c) e possibilita a emissão de relatórios indicativos do nível de ruído captado por cada coletor (celular) em um determinado período de tempo (Figura 3d).

Figura 3 - Prova de Conceito do sistema proposto

(a)

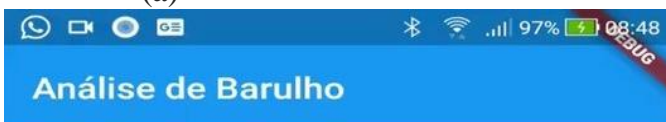

\begin{tabular}{c}
$\begin{array}{c}\text { Noise Level } \\
192.168 .86 .64\end{array}$ \\
MIC ON \\
ATUAL 47.38 \\
MAIOR 66.89 \\
Desconectar \\
\hline
\end{tabular}

(c)

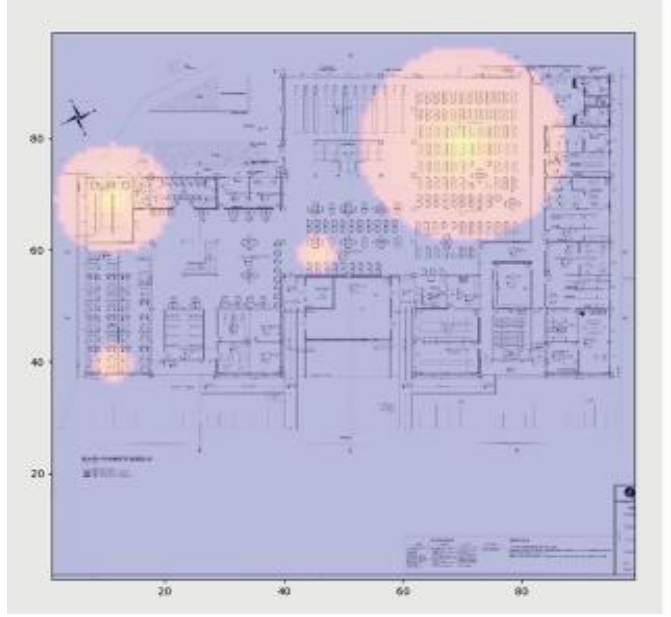

(b)

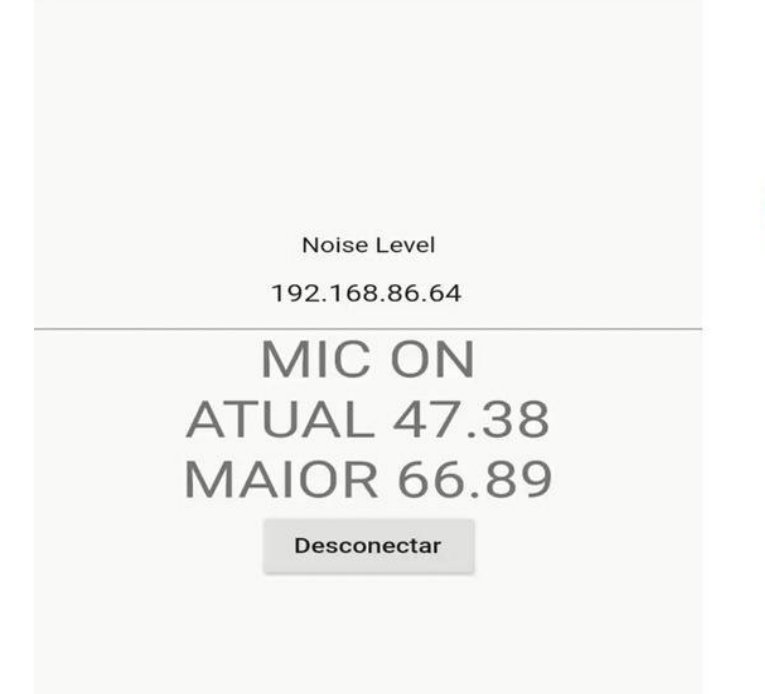

$\square$

Fonte: Imagens do sistema criado (2020). (d)

(1)

(b)
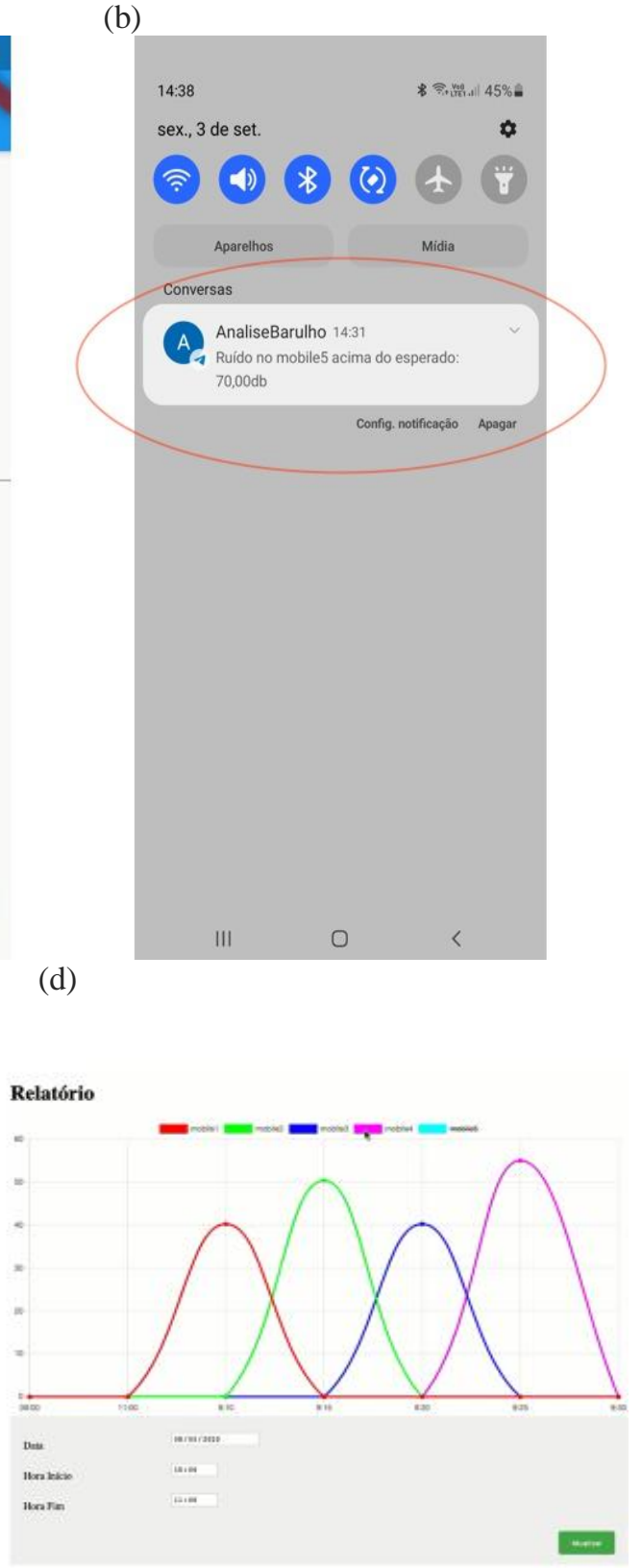
No PoC, foram implementadas as funcionalidades de captação de ruídos e geração simplificada de gráficos e mapa de calor para demonstrar a viabilidade técnica do projeto. Nesta primeira versão, foi emitido um sinal de alerta de ruído de alta intensidade pelo Bullet Push, um sistema para emissão de notificações. Para os usuários, pensou-se em utilizar a porta USB ou saída do fone de ouvido para acoplar um LED, que acenderia na mesa quando os usuários estivessem com um tom de voz muito alto.

\subsection{Avaliação do sistema proposto}

$\mathrm{Na}$ última fase da pesquisa, o sistema proposto foi avaliado pelos gestores, um profissional de TI e os usuários da BU/UFLA. Conforme os dados coletados, o DRS foi alterado para incorporar as sugestões de melhorias feitas pelos participantes da pesquisa e a implantação prática do sistema, testada pelo PoC, foi repensada.

\subsubsection{Avaliação do DRS}

Em relação à facilidade de entendimento do DRS, o documento foi considerado possuir uma linguagem clara e objetiva, que permitiu compreender o que se espera do sistema proposto e como se dá o seu funcionamento. Um único ponto de confusão na escrita do documento foi a utilização do termo "tendência" para referir-se aos dias e horários de pico de ruído dentro da biblioteca. Um dos participantes da pesquisa alertou que esse termo dentro da área de Computação é utilizado para referir-se à capacidade de previsão de um sistema. Por isso, o conceito mais adequado a ser utilizado no DRS é "histórico", pois trata-se de uma informação baseada em dados passados e não em uma projeção futura.

$\mathrm{O}$ produto Xi foi considerado excelente para lidar com o problema de excesso de ruído dentro da BU e criar um ambiente mais adequado para favorecer a concentração e foco nos estudos. Foi apontado que o bibliotecário terá facilidade para identificar a fonte do excesso de ruído e operacionalizar a tarefa de educar os usuários para adotarem um tom de voz mais ameno. 
Uma das bibliotecárias participantes da pesquisa afirmou ter recebido relatos de colegas de trabalho de outras instituições que possuem problemas similares em relação a barulho dentro de suas respectivas bibliotecas e por isso o sistema seria interessante para outras instituições, além da UFLA.

Uma expectativa com a implementação do sistema é que o próprio usuário perceba que está gerando muito barulho e tenha a consciência de adequar seu tom de voz ao ambiente em que se encontra. Mas, mesmo que seja necessário a intervenção de um bibliotecário, o fato de haver uma "comprovação" da fonte geradora de alto ruído faz com que os servidores encarregados da BU se sintam menos constrangidos por abordar os usuários.

Os requisitos descritos foram considerados bem especificados, mas foram sugeridas algumas alterações no DRS. Primeiramente, incluir um requisito sobre a manutenção do sistema para quando houver mudanças no layout da BU. Ou seja, sempre que o espaço físico for modificado, será necessário alterar o mapa virtual da biblioteca para mostrar o reposicionamento do mobiliário e dos celulares de captação de som. Para essa finalidade foi incluído no documento o requisito "Tela de Configuração", que fará parte da interface administrativa do sistema.

Foi sugerido também excluir do DRS a solução tecnológica testada pelo PoC, deixando em aberto essa questão, de forma a permitir que mais de uma solução seja testada antes da implementação do sistema na BU/UFLA. Essa questão será abordada mais detalhadamente no item 4.3.2. Por isso, optou-se por deixar registrado no DRS apenas uma indicação do que se espera da tecnologia utilizada, como por exemplo que seja de baixo custo e com baixo consumo de energia, sem especificação dos recursos tecnológicos.

Discutiu-se sobre inserir requisitos de desempenho relativos ao tempo de operação de algumas funcionalidades do sistema, como, por exemplo, a geração de relatórios. Entretanto, nos casos de relatórios muito longos, podem ser necessários vários minutos para finalizar a operação.. Por isso, julgou-se que o melhor seria realizar alguns testes com o sistema já pronto antes de especificar o tempo das operações. 
Os gestores e usuários concordaram que a decisão sobre a parametrização fixa do nível de ruído aceitável deve ser estabelecida após um período de testes. Posteriormente ao monitoramento por um determinado período de tempo e comparação com as normas de conforto acústico, a BU terá condições de determinar a partir de qual intensidade um ruído será considerado excessivo. Ademais, os parâmetros para disparar o alerta de ruído devem ser diferenciados para as áreas de estudo individual e coletiva.

Algumas questões consideradas relevantes pelos gestores da BU no momento de implementação do sistema:

a) necessidade de haver um equilíbrio entre o processo educativo de pedir colaboração dos usuários para manter o espaço com uma acústica confortável ao estudo,e ainda manter um diálogo amigável e aberto com os usuários;

b) frisar que não haverá gravação de voz, apenas captação dos decibels de som;

c) inicialmente, pode haver desconfianças em relação ao sistema e reclamações de alguns usuários sobre a falta de liberdade para conversar. Por isso, os servidores da BU devem estar preparados para uma fase de adaptação, onde os usuários se tornarão mais familiarizados com o sistema, aprenderão a moderar o tom de voz e se sentirão acostumados com a presença do sistema;

d) a divulgação do sistema deve focar nos benefícios a serem alcançados, deixando claro que a demanda pelo silêncio advém dos próprios usuários que frequentam o espaço, como comprovado na fase de especificação de requisitos;

e) a funcionalidade de medir a ocupação da biblioteca por meio do ruído foi vista como uma hipótese a ser testada, pois quando há poucos usuários o nível de ruído pode ficar tão baixo que o sistema não conseguirá detectar a presença de pessoas.

A funcionalidade do mapa de calor foi considerada "muito boa" pelos usuários durante a pesquisa. Esse mapa poderia ser visualizado em uma TV na 
entrada da biblioteca e também por meio do aplicativo de celular, de forma que os usuários possam consultar o mapa de calor independentemente de onde estejam. Contudo, foi questionado se o tamanho da tela do celular prejudicaria a visualização do mapa. Por isso, foi incluído no DRS um requisito para que o mapa possa ser ampliado na tela do celular para melhor visualização. Os usuários também demonstraram preocupação se o sistema realmente conseguiria fornecer informação em tempo real sobre as condições de ruído da biblioteca dado que a rede Wi-Fi da UFLA não é avaliada como muita rápida, como já verificado na análise dos dados dos questionários online com os usuários da BU/UFLA.

Os usuários reforçaram durante a coleta de dados a preferência por um sistema integrado a outros aplicativos disponibilizados pela UFLA, sugerindo que o Xi seja disponibilizado pela Minha UFLA (o aplicativo oficial da universidade). Por isso, adicionou-se ao DRS que o sistema deve ser desenvolvido como um serviço para permitir que seja acessado tanto por meio de um aplicativo de celular, pelo website da biblioteca, quanto integrado ao Minha UFLA.

Por fim, a adoção de novas tecnologias foi considerada importante para criar uma imagem institucional positiva da BU/UFLA, promovendo-a como uma entidade que busca constantemente melhorar a qualidade dos serviços oferecidos. Ademais, a incorporação de um recurso tecnológico pode, futuramente, abrir portas para outras possibilidades. Por exemplo, utilizar os mesmos celulares que realizam a captação de ruídos para medir a temperatura dos espaços da BU.

Após a análise das sugestões e considerações dos gestores, profissional de TI e usuários da BU/UFLA, o DRS foi revisado e aprimorado para incorporar as modificações pertinentes.

\subsubsection{Avaliação do PoC}

Em relação ao $\mathrm{PoC}$, surgiram algumas dúvidas em relação às tecnologias utilizadas e instalação dos dispositivos para captura dos decibels. As maiores preocupações foram: como os celulares serão fixados nas mesas e quanto tempo 
a bateria destes dispositivos duram (considerando que são aparelhos antigos, possivelmente já desgastados).

Foram feitas considerações acerca da segurança dos equipamentos instalados para captação do som, considerando que a universidade já teve registros de furtos de equipamentos que estavam aparafusados às salas de aula. O local de instalação dos celulares nas mesas é um ponto sensível para obter uma boa captação do som, mas não deve ocupar um espaço grande das mesas, já que os usuários necessitam de espaço para espalhar cadernos, livros e outros materiais de estudo.

A maior preocupação em relação a duração das baterias é que parte do sistema de captação de ruídos deixe de funcionar durante o horário de expediente, gerando inconsistências nos relatórios que serviriam de insumo à tomada de decisão dos gestores da BU. Esse receio advém principalmente do fato de que a antiga catraca eletrônica da BU parava de funcionar em alguns momentos, alterando significativamente as estatísticas sobre a entrada de alunos no recinto e deixando os relatórios de frequência de alunos na BU incoerentes com a realidade.

A ideia do power bank foi considerada pouco funcional. Primeiramente, porque gerará um custo extra para compra e possivelmente troca desses dispositivos após algum tempo de uso. Ademais um sistema com conexão direta à rede elétrica seria mais eficiente, pois limitaria a possibilidade de falhas no monitoramento e não exigiria a logística de realizar a troca de todos os power bank periodicamente. Mesmo que seja uma manutenção a cada semana ou mês, a BU possui mais de 100 mesas de estudo, o que demandaria um tempo longo para realizar a tarefa.

Em relação ao celular, foi sugerido que o dispositivo fique encoberto, pois esses aparelhos atualmente geram um sentimento de vigilância em algumas pessoas por ser de conhecimento a possibilidade desses aparelhos captarem conversas de forma não autorizada. Mesmo que a BU divulgue que o aparelho apenas coletará dados dos decibels dos ruídos do ambiente, a visão do celular pode gerar desconforto nos usuários. Outra preocupação é se os celulares 
captarão os decibels uniformemente, considerando que não serão da mesma marca e do mesmo modelo de fabricação.

Algumas alternativas surgiram durante a coleta de dados sobre tecnologias adequadas para implementar o sistema. A primeira é a possibilidade do Departamento de Ciência da Computação da universidade desenvolver dispositivos próprios, em substituição aos celulares. A segunda é instalar microfones cabeados para garantir a captação uniforme do som. O cabeamento poderia utilizar parte das canaletas já disponíveis no prédio da BU para armazenar os cabos elétricos e de rede. Contudo, é necessário testar se a eletricidade não interfere no som. Uma terceira ideia é utilizar um sistema baseado em triangulação de sinal que torne desnecessário a instalação de um dispositivo por mesa. Com a triangulação, um mesmo dispositivo seria capaz de identificar ruídos em raio de distância maior. Contudo, é necessário testar se a precisão para identificar as mesas individualmente não será prejudicada. Uma quarta sugestão foi utilizar os próprios celulares dos usuários para captar os sinais sonoros do ambiente. Para isso, seria necessário contar com o interesse dos usuários para participar de um projeto de economia colaborativa.

Dessa forma, concluiu-se que, para os testes iniciais de implantação do sistema, o celular é a melhor opção por já possuir diversos sensores. Mas para a implantação final do sistema, seria adequado fazer uma comparação da confiabilidade e custo dos celulares e outros dispositivos antes de optar pela melhor solução tecnológica.

A despeito desses questionamentos, o grupo gostou do ideal de sustentabilidade evocado pelo projeto, pois várias pessoas de fato possuem um celular velho em casa que não está sendo utilizado. A possibilidade da troca do celular por abatimento nas multas na BU também é uma possibilidade, pois o regimento permite campanhas para regularização de multas. Por exemplo, já houve uma campanha de doação de agasalhos a serem enviados para instituições de caridade em troca do abatimento de multa.

Finalizando a coleta de dados, alguns participantes da pesquisa afirmaram que, inicialmente, consideram que o projeto poderia ser de difícil execução. Mas, após avaliarem o DRS e PoC, acreditam que as dificuldades de 
implantação podem ser contornadas e esperam que haja continuidade do plano de implementação prática do sistema.

\section{Sistema para monitoramento de ruídos}

A emergência da tecnologia de IoT e sua ampla utilização nos mais diversos segmentos da sociedade modificará profundamente o cotidiano das pessoas, da mesma forma que o surgimento da internet provocou mudanças profundas desde o seu surgimento no final da década de 1960. O propósito desta pesquisa foi contribuir na discussão sobre como as bibliotecas podem se inserir neste novo cenário da $4^{a}$ Revolução Industrial e aprimorar os serviços e produtos oferecidos aos seus usuários.

O fruto desse trabalho coletivo resultou no desenvolvimento de uma proposta de sistema para monitoramento de ruídos dentro da BU/UFLA, almejando criar recursos para auxiliar na promoção de um ambiente adequado para estudos e fornecer dados para a tomada de decisão dentro da biblioteca. Embora o sistema proposto tenha sido construído com base nas demandas específicas dos gestores e usuários da BU/UFLA, espera-se que o sistema Xi possa ser aplicado em diferentes ambientes informacionais. Pois, ao documentar o percurso metodológico, torna-se possível que outros pesquisadores ou bibliotecários sejam capazes de replicar a metodologia adotada para criar sistemas similares, customizados de acordo com as necessidades e limitações das instituições onde estão inseridos.

Recomenda-se para estudos futuros investigar diferentes soluções tecnológicas para a implementação do sistema Xi. É necessário testar opções que sejam viáveis financeiramente e sejam capazes de oferecer um produto eficiente, com o mínimo de falhas. Dessa forma, um projeto-piloto a ser implantado em uma área restrita da biblioteca oferecerá dados importantes antes de expandir o monitoramento de ruído para todas as áreas de estudo do edifício.

Conclui-se que a IoT oferece oportunidades de facilitar a rotina de trabalho dentro das bibliotecas e disponibilizar aos usuários um ambiente e atendimento mais adequado às suas necessidades. Por meio da coleta de dados, os bibliotecários terão à sua disposição informações sobre a utilização do acervo 
e espaço físico da biblioteca, além de serem capazes de compreender melhor o comportamento do público atendido. Esses dados constituirão um insumo valioso para a gestão da biblioteca e a tomada de decisões mais assertivas e baseadas em evidências.

Por sua vez, os usuários terão à sua disposição ambientes e serviços mais adaptáveis às suas necessidades individuais e com maior valor agregado. Por meio da IoT, a experiência de uso da biblioteca pode tornar-se mais intuitiva, ágil e agradável, atendendo demandas que talvez nem mesmo os usuários consigam identificar e expressar explicitamente.

\section{Referências}

ANDRADE, R. M. de; ARAKAKI, R.; BECERRA, J. L. R. O uso de Provas de Conceito como ferramenta para gestão de aprendizado de arquitetura de software. In: CONGRESSO INTERNACIONAL DE GESTÃO DA TECNOLOGIA E SISTEMAS DE INFORMAÇÃO, 3., 2006, São Paulo. Anais [...]. São Paulo: USP 2006. p. 284-300.

ASHTON, K. That 'Internet of Things' thing. RFID Journal, Alpharetta, 22 jun. 2009.

\section{ASSOCIAÇÃO BRASILEIRA DE NORMAS TÉCNICAS (ABNT). NBR}

10152: níveis de pressão sonora em ambientes internos a edificações. São Paulo: ABNT, 2017. Versão corrigida em 2020.

ATZORI, L.; IERA, A.; MORABITO, G. The Internet of Things: a survey. Computer Networks, [Amsterdam], v. 54, n. 15, p. 2787-2805, Oct. 2010.

BARBETTA, P. A. Estatística aplicada às ciências sociais. 5. ed. Florianópolis: Ed. da UFSC, 2002.

BOTARI, J. C. et al. Análise do Conforto Acústico em Biblioteca da Universidade Estadual de Maringá do Campus Umuarama - PR. In: SAFETY, HEALTH AND ENVIRONMENT WORLD CONGRESS, 17., 2017, Vila Real. Proceedings [...]. Vila Real: SHEWC, 2017. p. 115-119.

CÂNDIDO, A. L. M. et al. Low cost device for online monitoring of noise in libraries using internet of things. International Journal for Innovation Education and Research, Dhaka, v. 6, n. 8, p. 133-144, 2018.

CÂNDIDO, A. L. M.; JUCÁ, S. C. S. Monitoramento online de ruídos sonoros de bibliotecas utilizando o princípio internet das coisas. In: ESCOLA 
REGIONAL DE INFORMÁTICA DO PIAUÍ, 3., 2017, Picos. Anais [...]. Teresina: UFPI, 2017. p. 147-152.

COLUMBUS, L. Seven Things You Need To Know About IIoT In Manufacturing. Forbes, [S. l.], 2 Jun. 2019.

DOMINGO, M. C. An overview of the Internet of Things for people with disabilities. Journal of Network and Computer Applications, [Amsterdam], v. 35, n. 2, p. 584-596, Mar. 2012.

DUTRA, M. L.; TORIANI, S. A Internet das Coisas na prática: desafios e oportunidades. In: PRADO, J. do (Org.). Ideias emergentes em

Biblioteconomia. São Paulo: FEBAB, 2016, p. 1136-1147.

FREITAS, H.; JANISSEK, R. Análise léxica e análise de conteúdo: técnicas complementares, sequenciais e recorrentes para análise de dados qualitativos. Porto Alegre: Sphinx, 2000.

HAHN, J. The Internet of Things: Mobile Technology and Location Services in Libraries. In: AMERICAN LIBRARY ASSOCIATION (ALA). Library

Technology Reports: Expert Guides to Library Systems and Services, Chicago, v. 53, n. 1, Jan. 2017.

\section{INSTITUTE OF ELECTRICAL AND ELECTRONICS ENGINEERS. IEEE}

Std 830-1998: IEEE recommended practice for software requirements specifications. Nova York: IEEE, 1998. Disponível em:

http://www.math.uaa.alaska.edu/ afkjm/cs401/IEEE830.pdf. Acesso em: 13 jul. 2020 .

KHAN, R. et al. Future internet: the internet of things architecture, possible applications and key challenges. In: INTERNATIONAL CONFERENCE ON FRONTIERS OF INFORMATION TECHNOLOGY, 10., 2012, Islamabad. Proceedings [...]. [Bremen: IEEE], 2012. p. 257-260.

MACWHINNIE, L. A. The Information Commons: The Academic Library Of The Future. Portal: Libraries and the Academy, Baltimore, v. 3, n. 2, p. 241257, Abr. 2003.

MEDEIROS, A. P. et al. Detector de ruídos aplicado a ambientes de estudo. In: CONGRESSO NORTE NORDESTE DE PESQUISA E INOVAÇÃO, 8., 2013, Salvador. Anais [...]. Salvador: IFBA, 2013.

ONLINE COMPUTER LIBRARY CENTER (OCLC). The internet of things: 50 billion connected devices and objects by the year 2020. Next Space, Dublin, n. 24, Jan. 2015. 
OCCUPATIONAL SAFETY AND HEALTH ADMINISTRATION.

Monitoring noise levels non-mandatory informational apêndix. Washington, DC: OSHA, 1996.

PEREIRA, C. A. S.; SILVA, L. C. S.; SALES, F. H. S. Análise do nível de conforto acústico na biblioteca de uma escola pública. Holos, Natal, v. 27, n. 4, p. 65-91, 2011.

REIS, E. Estatística descritiva. 7. ed. Lisboa: Silabo, 2008.

ROCHA, R. et al. Efeito de estresse ambiental sobre a pressão arterial de trabalhadores. Revista de Saúde Pública, São Paulo, v. 36, n. 5, p. 568575, out. 2002.

SANTOS, C. M. L. da S. A. dos. Estatística descritiva: manual de autoaprendizagem. 3. ed. Lisboa: Sílabo, 2018.

SCULLY, P. IIOT platforms for manufacturing 2019-2024. Hamburg: IoT Analytcs GmbH, 2019.

SERVILHA, E. A. M.; DELATTI, M. de A. Percepção de ruído em sala de aula por estudantes universitários e suas consequências sobre a qualidade do aprendizado. Audiology - Communication Research, São Paulo, v. 19, n. 2, p. 138-144, abr./jun. 2014.

SHIN, D. H. A realization of pervasive computing: ubiquitous city. In: TECHNOLOGY MANAGEMENT FOR GLOBAL ECONOMIC GROWTH, 2010, Phuket. Proceedings [...]. [New Jersey: IEEE], 2010. p. 1-10.

SILVA, R. C.; ALMEIDA, M. das N.; ALBUQUERQUE NETO, H. C. Análise do nível de pressão sonora em uma biblioteca de uma instituição de ensino superior. In: ENCONTRO NACIONAL DE ENGENHARIA DE PRODUÇÃO, 37., 2017, Joinville. Anais [...]. Rio de Janeiro: Associação Brasileira de Engenharia de Produção, 2017. p. 1-19.

ZANELLA, A. et al. Internet of Things for Smart Cities. IEEE Internet Of Things Journal, [S. l.], v. 1, n. 1, p. 22-32, Feb. 2014.

\title{
Internet of things in libraries: a noise monitoring system proposal for libraries
}

\begin{abstract}
Libraries are information units that are constantly adopting new technological resources to improve the quality of their offer of products and services. Currently, a prominent technology is the internet of things, which
\end{abstract}


promises major changes in society by providing greater integration between the physical and digital worlds. Therefore, this study aims to discuss the possibility of implementing IoT in a university library by investigating the demands of users that could be addressed through this technology. It is an applied research, characterized as exploratory and descriptive. The methodology adopted is a case study at the University Library of the University of Lavras, based on requirements engineering and design thinking. The final result is the idealization of a noise monitoring system, based on IoT, whose purpose is to contribute to the maintenance of silence in the study areas and the use of noise as a data to support the library management.

Keywords: Internet of Things; Noise Monitoring System; Library Management; Smart Libraries

Recebido: $24 / 11 / 2020$

Aceito: 07/05/2021

\section{Declaração de autoria}

Concepção e elaboração do estudo: F. V. Amaral, J. P. Juliani, R. W. de Bettio

Coleta de dados: F. V. Amaral

Análise e interpretação de dados: F. V. Amaral, J. P. Juliani, R. W. de Bettio

Redação: F. V. Amaral, J. P. Juliani, R. W. de Bettio

Revisão crítica do manuscrito: F. V. Amaral, J. P. Juliani, R. W. de Bettio

\section{Como citar}

AMARAL, Fernanda Vasconcelos; JULIANI, Jordan Paulesky; BETTIO, Raphael Winckler de. Internet das coisas em bibliotecas: proposta de um sistema para monitoramento de ruído para bibliotecas. Em Questão, Porto Alegre, v. 28, n. 1, p. 458-483, 2022. DOI: http://dx.doi.org/10.19132/1808$5245281.458-483$ 